\title{
Investigation of acceptor levels and hole scattering mechanisms in p-gallium selenide by means of transport measurements under pressure
}

\author{
D.Errandonea ${ }^{\dagger}$, J.F.Sánchez-Royo and A.Segura \\ Institut de Ciència dels Materials, Universitat de València \\ Dpto. de Física Aplicada, Ed. Investigació, Univ. de València, \\ C/Dr. Moliner 50, E-46100 Burjassot (València), Spain
}

\begin{abstract}
A.Chevy and L.Roa
Laboratoire de Physique des Mileux Condensés, Univ. Pierre et Marie Curie, 4 place Jussieu, 75252 Paris, Cedex 05, France
\end{abstract}

\begin{abstract}
The effect of pressure on acceptor levels and hole scattering mechanisms in $\mathrm{p}$-GaSe is investigated through Hall effect and resistivity measurements under quasi-hydrostatic conditions up to $4 \mathrm{GPa}$. The pressure dependence of the hole concentration is interpreted through a carrier statistics equation with a single (nitrogen) or double (tin) acceptor whose ionization energies decrease under pressure due to the dielectric constant increase. The pressure effect on the hole mobility is also accounted for by considering the pressure dependencies of both the phonon frequencies and the hole-phonon coupling constants involved in the scattering rates.
\end{abstract}

Keywords: GaSe, High pressure, Transport properties, Acceptor levels, and Scattering mechanisms.

Short title: Transport properties of p-GaSe under pressure.

${ }^{\dagger}$ Corresponding author, E-mail: daniel@ges1.fisapl.uv.es, Fax: 3463983146. 


\section{INTRODUCTION}

Gallium selenide (GaSe) belongs to III-VI layered semiconductor family which is characterized by a strong anisotropy in the chemical bonding. Its crystal structure consists of plane hexagonal lattices which are associated in pairs and may be stacked in different ways $(\beta, \gamma, \varepsilon$-polytypes)[1]-[2], being the most common one the corresponding to $\varepsilon$-polytype ( $D_{3 h}^{1}$ space group). The possibility of growing III-VI semiconductors thin films by van der Waals epitaxy [3]- [6] opens new opportunities to their potential practical applications, e.g. electronic devices [5] and solar cells[7]. In this respect, the study of the fundamental electrical transport properties is a very important issue. In the case of GaSe, at ambient pressure, these properties have been investigated in detail by different authors who have focused their attention on the role played by impurities such as $\mathrm{Cd}[8], \mathrm{Cu}[9], \mathrm{N}[10], \mathrm{Sn}[1]$ and $\mathrm{Zn}[12$. In contrast, very little is known about the behaviour of transport properties under pressure $(\mathrm{P})$.

Pressure experiments are an efficient tool to study III-VI semiconductors since they allow the tuning of the degree of anisotropy of these materials. Experiental studies of their optical [13]-[17] and lattice dynamical[17]-20 properties under pressure have been subjects of recent interest. However, systematic research of the electrical transport properties under compression has been only made for indium selenide (InSe), for which, resistivity $(\rho)$ and Hall effect (HE) measurements under pressure have been reported very recently[21].

In this article, we report a systematic study on transport properties under high pressure, up to $4 \mathrm{GPa}$ at room temperature (RT), of the III-VI layered p-GaSe. In Section II we briefly describe the experimental setup. The data obtained on HE and resistivity measurements are presented in Section III. Finally, Section IV is devoted to the discussion of these results and their implications.

\section{EXPERIMENT}

The p-GaSe single crystals used in this study were grown by the conventional 
Bridgman technique. Doping by tin (Sn) was performed by adding the pure element so as to get 0.05, 0.1, 0.2 and 0.5 at \% in the stoichiometric melt of GaSe. Nitrogen $(\mathrm{N})$ was introduced as GaN compound in a quantity to give a concentration of 0.5 at \% of $\mathrm{N}$ in the melt. We should point out that these concentrations are different from the effective ones, since during the Bridgman growth most of the initial impurity concentration is segregated to the end of the ingot [22].

Samples with faces perpendicular to the c-axis were prepared from the ingots by cleaving and cutting with a razor blade. Thickness of the slabs was measured by using the interference fringe pattern in the infrared transmission spectrum. Typical sample dimensions were $70 \mu \mathrm{m}$ in thickness and about $4 \times 4 \mathrm{~mm}^{2}$ in size. Pressure up to $4 \mathrm{GPa}$ was applied to the samples by using the Bridgman cell that has been described in Reference [21]. In this case, we have used tungsten carbide anvils, $27 \mathrm{~mm}$ in diameter, which were put between the pistons of a 150-ton press. Sodium chloride was the pressure transmitting-medium. The pyrophillite gaskets were $0.5 \mathrm{~mm}$ in thickness, with a hole of $9 \mathrm{~mm}$. The Bridgman gasket assembly and geometry are shown in the inset of Figure 1. Ohmic contacts were made in a van der Pauw geometry[23] by soldering silver wires with high-purity indium in gold contact pads which were previously vacuum evaporated. HE and resistivity measurements were carried out at RT. Direct electric current ranging from $50 \mu \mathrm{A}$ to $500 \mu \mathrm{A}$ was sent through the samples, and the resulting voltages were measured by a digital voltmeter. The linearity of the ohmic voltages on the injected current was checked out at different pressures. A magnetic field of 0.6 Tesla was applied parallel to the c-axis. The magnetic field was generated by means of a copper coil placed around one of the pistons of the press.

\section{RESULTS}

Figure 1 shows the pressure dependence of resistivity for N-doped and Sn-doped GaSe samples. The nominal doping concentration $([\mathrm{N}]$ or $[\mathrm{Sn}])$ of each measured sample is also given in this figure. Resistivity appears to decrease with pressure in all the samples here studied. This evolution is more pronounced in the samples 
doped with $\mathrm{N}$, in which $\rho$ goes down a factor three in the explored range of pressure. This factor is nearly constant in all the Sn-doped samples, in which resistivity decreases with doping, except in the slabs with the highest tin content $([\mathrm{Sn}]=$ $0.5 \%)$.

The decrease of the resistivity with pressure turns out to be due to the increase of both the hole concentration and mobility, as shown in Figures 2 and 3. Figure 2 shows the pressure behaviour of the hole concentration $(p)$ for different samples as determined through

$$
p=\frac{1}{q R_{H}}
$$

where $q$ is the electron charge and $R_{H}$ is the Hall coefficient. The Hall factor has been assumed to be 1 10, 11]. It can be seen in Figure 2 that $p$ non-linearly increases under compression, the relative variation of $p$ being higher for samples doped with N. In samples doped with $\mathrm{Sn}$, at zero pressure $p$ is enlarged with increasing $[\mathrm{Sn}]$ with the exception of the $0.5 \%$ Sn-doped sample in which $p$ is two

orders of magnitude smaller. In addition, in this sample the relative change of $p$ is the largest among the Sn-doped samples.

As regards the mobility $(\mu)$, Figure 3 shows its pressure dependence. It can be seen there that the mobility also increases with pressure and that its value at ambient pressure is very similar to that obtained in previous works 10, 11].

\section{DISCUSSION}

\subsection{Pressure Dependence of the Hole Concentration}

To understand the pressure dependence of $p$ we have considered that N-doped and Sn-doped GaSe at RT are extrinsic as reflected in the temperature dependence of their transport properties]10, 11]. Moreover, from the temperature behaviour of the hole concentration it was deduced that doping by $\mathrm{N}$ introduces only one acceptor level with ionization energy of $E_{a}=210 \mathrm{meV}[10]$. In the Sn-doped samples two impurity levels with ionization energies of $E_{a}=155$ and $310 \mathrm{meV}$ have been observed [11]. These levels appear to be connected with the presence of a double 
acceptor-impurity. This acceptor has been proposed to be an interstitial Sn atom in the octahedral interlayer site associated to a close Ga-vacancy, giving a local configuration similar to that of $\mathrm{Sn}$ in the layered compound $\mathrm{SnSe}_{2}$ [11]. At RT the first level $\left(E_{a}=155 \mathrm{meV}\right)$ is not fully ionized, so that the pressure dependence of $p$ is mainly determined by its behaviour under pressure. However, when increasing the doping of Sn to $0.5 \%$ a decrease of the hole concentration has been observed (see Figure 2) indicating that, at very high Sn concentration, a donor configuration of Sn in GaSe (isolated Sn interstitial or Sn substituting to Ga) becomes dominant and overcompensates the acceptor centers. Then, as in the heaviest doped samples the first level $(155 \mathrm{meV})$ is completely compensated, the behaviour of the second level (310 meV) would determine the variation of $p$ under compression. Therefore, we can analyze in both cases the pressure dependence of $p$ through a single acceptor-single donor model for partially compensated p-type semiconductors [24]. Within this model the hole concentration is given by:

$$
\begin{aligned}
p= & \frac{N_{d}}{2}\left\{-1-\frac{N_{v}}{2 N_{d}} \exp \left(\frac{-E_{a}}{k T}\right)+\right. \\
& {\left.\left[1+\frac{N_{v}}{N_{d}} \exp \left(\frac{-E_{a}}{k T}\right)\left(-1+\frac{N_{v}}{4 N_{d}} \exp \left(\frac{-E_{a}}{k T}\right)+\frac{2 N_{a}}{N_{d}}\right)\right]^{1 / 2}\right\} }
\end{aligned}
$$

where $T$ is the absolute temperature, $k$ is the Boltzmann constant, $E_{a}$ refers to the ionization energy of the acceptor level, $N_{d}$ and $N_{a}$ are the donor and acceptor impurity concentration, respectively, and $N_{v}$ is the density of states of the valence band which can be written at RT as a function of the effective mass in the valence band $m_{v}^{*}=\left(m_{h \perp}^{*} m_{h \|}^{*}\right)^{1 / 3}$ as:

$$
N_{v}=2.509 \times 10^{19}\left(\frac{m_{v}^{*}}{m_{o}}\right)^{3 / 2} \mathrm{~cm}^{-3},
$$

where $m_{o}, m_{h \perp}^{*}$, and $m_{h \|}^{*}$ stand for the free electron mass, the perpendicular and parallel effective hole masses, respectively. Taking $m_{h \perp}^{*}=0.8 m_{o}$ and $m_{h \|}^{*}=0.2$ $m_{o}$ at zero pressure [25, 26], we obtain $m_{v}^{*}=0.5 m_{o}$.

In Eq.(2) one can see that $p$ depends on the pressure through $m_{v}^{*}, E_{a}$ and the term $N_{d} / 2$. Following the Kane model[27], the variation of the effective mass with 
pressure is considered to be proportional to the change of the direct band-gap [15]. For the pressure dependence of $N_{d}$ it can be assumed that it is determined by the volume variation[17]. Then, taking for $N_{a}, N_{d}$ and $E_{a}$ at ambient pressure the values deduced from the temperature dependence of the hole concentration [10, 11], and using Eq.(2), we can calculate $E_{a}$ at each pressure. The result is shown in Figure 4 (points). There it can be seen that the observed behaviour of $p$ would be a consequence of a reduction of the ionization energies under pressure. For the same pressure variation (from 0 to $4 \mathrm{GPa}$ ) the ionization energy of the N-related acceptor would decrease by $20 \%$, but that of the Sn-related one, only by 6.4 or $8.1 \%$.

The ionization energy of a single hydrogenic impurity level can be evaluated in an anisotropic crystal through the Gerlach-Pollman model[28]. By using the effective masses of GaSe from References [25, 26] and the static dielectric constant at ambient conditions from Reference [17 one obtains a value of $E_{a}=72 \mathrm{meV}$. The discrepancy between this value and the experiment can be attributed to the differences between the Coulomb potential used in the hydrogenic model and the true impurity potential. It has been shown that the true impurity potential can be represented by an effective potential which includes a central-cell correction to the Coulomb potential model[29]. Then, to modelize the pressure dependence of $E_{a}$ we take the impurity potential to be[29]:

$$
V(r)=-\frac{2 Z R^{*} a^{*}}{r}[1-\exp (-b r)+B r \exp (-b r)] f(\alpha, \theta),
$$

where $R^{*}, a^{*}$ and $f(\alpha, \theta)$ are the effective Rydberg energy, the effective Bohr radius and the anisotropy function defined in the Gerlach-Pollman model, respectively, $b$ and $B$ are the adjustable potential parameters, and $Z$ is equal to $1(2)$ for the single(double) ionized acceptor-impurity. At small $r$, this potential looks like a sphericall well, with a depth $V_{0}$ given by:

$$
V_{0}=-q^{2} \frac{B+b}{\varepsilon_{0 \perp} \varepsilon_{0 \|}},
$$

where $\varepsilon_{0 \perp}$ and $\varepsilon_{0 \|}$ are the static dielectric constant in the direction perpendicular and parallel to the c-axis, respectively. At large $r$ it behaves like a screened 
Coulomb potential. The turning point is given approximately by

$$
r_{z}=\frac{B+b}{B b}
$$

The proposed impurity potential model and the Coulomb potential are shown schematically in the inset of Figure 4.

The Schrödinger equation of the system is solved by the variational method by using a trial function for the electron ground state $\left(\Psi_{0}(r)\right)$ as:

$$
\Psi_{0}(r)=\sqrt{\frac{b^{3}}{\pi}} \exp (-b r)
$$

The variational calculation in which the total energy is minimized leads to:

$$
E_{a}=-\left(\frac{5}{9}\right)^{2} R^{*} Z_{0}^{2}(\alpha)-\frac{16}{27} B R^{*} a^{*} Z_{0}(\alpha)
$$

where $Z_{0}(\alpha)$ is the effective charge 28]. Then the potential parameter $B$ is chosen to fit the ionization energy of the single acceptor level at room pressure. The fitted value of $B$ is shown in Table 1 together with $V_{0}$ and $r_{z}$. $E_{a}$ depends on the pressure through $R^{*}$ and $a^{*}$. The first term of Eq.(7) decreases under pressure due to the reduction of $R^{*}$ as a consequence of the increase of $\varepsilon_{0 \|}$ [17, 30]. The second term also decreases under pressure, but more slowly because $R^{*} a^{*} \sim\left(\varepsilon_{0 \|}\right)^{-1 / 2}$.

The calculated pressure dependence of $E_{a}$ is shown in Figure 4 (solid lines). It can be seen there that the model fits quite well the experiment. The shift observed in the second Sn-related level may be connected with the fact that in the present model we have neglected the electron-electron interactions and, as a consequence of this, we could be overestimating the value of $E_{a}$. To understand why $E_{a}$ suffers a greater decrease in $\mathrm{N}$-doped samples, let us consider the parameters given in Table 1. It can be seen there that in this case the square well is deeper. This is just what one may expect because $\mathrm{N}$ is much more electronegative than $\mathrm{Sn}$. That is why the central-cell correction is more important in N-doped samples than in Sn-doped samples. Because of this a deeper acceptor level is obtained. In addition, the second term of Eq.(7) would decrease more rapidly leading to the greater change observed in $E_{a}$. 


\subsection{Pressure Dependence of the Hole Mobility}

Ionized impurity scattering and two-phonon scattering mechanisms must be considered in order to give a quantitative account of the pressure dependence of the hole mobility. The ionized impurity concentration has been assumed to be that obtained from the temperature dependence of the hole concentration [10, [1]]. The phonons involved are the $138 \mathrm{~cm}^{-1} A_{1}^{\prime 2}$ homopolar optical mode and the 153.2 $\mathrm{cm}^{-1} E^{\prime 3}$ LO polar optical mode [17. The pressure dependence of the Fröhlich constant [3] $(\alpha)$ and the hole-homopolar phonon coupling constant 32] $\left(g^{2}\right)$ were calculated from data found in the literature[17, 33, 34]. As the hole scattering rate for LO phonons is obtained through an integration over all the possible directions of the phonon momentum, $\alpha$ was calculated through [35:

$$
\alpha=\frac{2}{3} \alpha_{\perp}+\frac{1}{3} \alpha_{\|} .
$$

We have obtained for both coupling constants a decreasing behaviour under pressure. The reduction of $g^{2}$ is basically a result of the increase of the homopolar phonon frequency under pressure [17, [18]. For $\alpha$, in spite of the increase of $\alpha_{\|}$, due to the great increase of $\varepsilon_{0 \|}$ under compression [17, 30], the overall change is negative because of the decrease of $\alpha_{\perp}$, which is more than four times higher than $\alpha_{\|}$

By means of an iterative method 24] we have calculated the pressure dependence of $\mu$. The ionized impurity scattering has been included through the Brooks-Herring relaxation time [36], in which the ionized impurity concentrations has been assumed to be that used to calculate $E_{a}$ a a function of pressure. The results obtained for the samples doped with $[\mathrm{Sn}]=0.05 \%$ and $0.1 \%$ and with $[\mathrm{N}]=0.5 \%$, which are plotted in solid lines in Figure 3, agree quite well with the measurements. The isolated contribution of each scattering mechanism is also represented in Figure 3. The homopolar phonon scattering (curve 1) and the LO polar phonon scattering (curve 2) are the dominant mechanisms over the whole pressure range, but the ionized impurity scattering mechanism (curve 3) must be taken into account to reproduce quantitatively the absolute value of $\mu$. The saturation of the increase of $\mu$ above $2.5 \mathrm{GPa}$ is due to the saturation of the decrease of $\alpha$ as a consequence of 
the compensation between the LO polar phonon frequency and $\varepsilon_{0 \|}$ increases. In addition, the increase of the concentration of ionized impurities, determined by the increase of $p$ and the contraction of the volume under compression, leads to a reduction of the impurity-limited mobility, which also collaborates to the saturation of $\mu$.

Finally, we want to point out that for samples doped with $[\mathrm{Sn}]=0.2 \%$ and $0.5 \%$ the value of the zero pressure mobility cannot be reproduced with the present model. Samples from the $0.2 \%$ ingot exhibit a phonon-controlled mobility in a larger temperature range than those from ingots with lower tin content. We think that this can be related to the fact that a large proportion of complex impurity centers are present in these samples, resulting in a reduction of the concentration of single ionized impurities 11]. These complex centers appear to have a lower scattering cross section than ionized impurities, producing that scattering by phonons is the dominant scattering mechanism in spite of the heavy doping concentration of the $0.2 \%$ Sn-doped sample. Nevertheless, the structure of those centers and its influence on the hole mobility is not known and can hardly be included in our model. In samples from the $0.5 \%$ Sn-doped ingot the compensation is very high and impurity scattering is dominant even at room temperature reducing the mobility to the low value observed $\left(\mu=9.5 \mathrm{~cm}^{2} / \mathrm{Vs}\right)$, but this value and its temperature dependence could not be accounted for through any known scattering mechanism.

\section{CONCLUSIONS}

Transport measurements have been carried out under pressure in N-doped and Sn-doped GaSe up to $4 \mathrm{GPa}$. Within the framework of a single acceptor-single donor model, the observed increase of $p$ under compression has been interpreted to be due to the reduction of $E_{a}$ with pressure. Modeling the impurity potential we have obtained an expression for $E_{a}$. This allows us to relate the decrease under pressure of $E_{a}$ to the increase of $\varepsilon_{0 \|}$. In addition, we have also discussed the different behaviour under pressure of the ionization energies of the acceptor 
levels connected to Sn and $\mathrm{N}$ as doping impurities in GaSe. The higher reduction of $E_{a}$ observed in $\mathrm{N}$-doped samples has been explained by means of the deeper central-cell correction obtained in this case. The observed increase of $\mu$ has been attributed to the decrease of the hole-phonon coupling constants.

\section{ACKNOWLEDGMENTS}

This work was supported by the Spanish Government CICYT under Grant No: MAT $95-0391$.

\section{References}

[1] J.C.J.R.Terhell and R.M.A.Lieth, Phys.Stat.Sol.(a) 5, 719 (1971).

[2] A.Kuhn, R.Chevalier, and A.Rimsky, Acta Cryst.Sect. 31, 2841 (1975).

[3] A.Koma, Thin Solid Films 216, 72 (1992).

[4] W.Jaegermann, Photoelectrochemestry and photovoltaics of layered semiconductors, edited by A.Aruchamy (Kluwer, Dordrecht, 1992), pp. 195-295.

[5] O.Lang, R.Schlaf, Y.Tomm, C.Pettenkofer, and W.Jaegermann, J.Appl.Phys. 75, 7805 (1994).

[6] O.Lang, R.Schlaf, Y.Tomm, C.Pettenkofer, and W.Jaegermann, J.Appl.Phys. 75, 7814 (1994).

[7] A.Segura, J.M.Besson, A.Chevy, and M.S.Martin, Nuovo Cimento 38, 345 (1977).

[8] S.Shigetomi, T.Ikari, and H.Nishimura, J. Appl. Phys. 69, 7936 (1991).

[9] V.Capozzi, Phys.Rev. B 28, 4620 (1983).

[10] J.F.Sánchez Royo, A.Segura, A.Chevy, and L.Roa, J. Appl. Phys. 79, 204 (1996).

[11] J.F.Sánchez Royo et al (unpublished).

[12] S.Shigetomi, T.Ikari, and H.Nishimura, J. Appl. Phys. 74, 4128 (1993).

[13] J.M.Besson, K.P.Jain, and A.Kuhn, Phys.Rev.Lett. 32, 936 (1974).

[14] M.Mejatty, A.Segura, R.Le Toulec, J.M.Besson, A.Chevy, and H.Fair, J. Phys. Chem. Solids 39, 25 (1978).

[15] N.Kuroda, O.Ueno, and Y.Nishina, J.Phys.Soc.Jpn. 55, 581 (1986).

[16] A.R.Goñi, A.Cantarero, U.Schwarz, K.Syassen, and A.Chevy, Phys.Rev. B 45, 4221 (1992). 
[17] M.Gauthier, A.Polian, J.M.Besson, and A.Chevy, Phys.Rev. B 40, 3837 (1989).

[18] N.Kuroda, O.Ueno, and Y.Nishina, Phys.Rev. B 35, 3860 (1987).

[19] C.Ulrich, M.A.Mogrinski, A.R.Goñi, A.Cantarero, U.Schwarz, V.Muñoz, and K.Syassen, Proceedings of the 7th International Conference of High Pressure Semiconductor Physics, edited by K.Syassen, A.R.Stradling, and A.R.Goñi, [Phys. Stat. Sol. (b) 198, 121 (1996)].

[20] A.Polian, J.C.Chervin, and J.M.Besson, Phys.Rev. B 22, 3049 (1980).

[21] D.Errandonea, A.Segura, J.F.Sánchez-Royo, V.Muñoz, P.Grima, A.Chevy, and C.Ulrich, Phys.Rev. B 55, 16217 (1997).

[22] A.Chevy, J.Appl.Phys. 56, 978 (1984).

[23] J.L.van der Pauw, Philips Research Report 13, 1 (1955).

[24] K.Seeger, Semiconductor Physics, Springer series in solid states sciences vol 40. [Springer - Verlag, Berlin Heidelberg, 1982].

[25] G.Ottavi, C.Canali, F.Nava, Ph.Schmid, E.Mooser, R.Minder, and I.Schokke, Solid State Commun. 14, 993 (1974).

[26] E.Mooser, and E.Schlüter, Il Nuovo Cimento b 18, 164 (1973).

[27] E.M.Conwell, High Field Transport in Semiconductors, [Academic, New York, 1972].

[28] B.Gerlach and J.Pollmann, Phys.Stat.Sol. (b) 67, 93 (1975).

[29] T.H.Ning and C.T.Sah, Phys.Rev B 4, 3468 (1971).

[30] D.Errandonea (unpublished)

[31] H.Fröhlich, Adv.Phys. 3, 325 (1954).

[32] R.Fivaz and E.Mooser, Phys.Rev. 163, 743 (1967).

[33] Ph.Schmid and J.Voitchousky, Phys.Stat.Sol. (b) 65, 249 (1974).

[34] G.Martinez, Handbook of Semiconductors, edited by J.Moss and M.Balkanski [Nort Holland, Amsterdam, 1980], vol. 2, p. 181.

[35] A.Segura, B.Marí, J.Martinez-Pastor, and A.Chevy, Phys.Rev. B 43, 4953 (1991).

[36] H.Brooks, Advances in Electronics and Electron Physics [Academic, New York, 1955], vol. 7, p. 85 . 
Table 1: Model parameter $B$, deep of the square well $V_{0}$ and turning point $r_{z}$.

\begin{tabular}{cccc}
\hline \hline Impurity & $B$ & $V_{0}$ & $r_{z}$ \\
\hline $\mathrm{Sn}_{1}$ & 0.36 & $-0.725 \mathrm{eV}$ & $4.40 a^{*}$ \\
$\mathrm{Sn}_{2}$ & 0.36 & $-1.120 \mathrm{eV}$ & $2.47 a^{*}$ \\
$\mathrm{~N}$ & 0.53 & $-1.020 \mathrm{eV}$ & $4.25 a^{*}$ \\
\hline \hline
\end{tabular}




\section{Figure captions}

Figure 1: Resistivity as a function of pressure for different samples. The inicial doping concentrations in the growth solutions $([\mathrm{N}]$ or $[\mathrm{Sn}])$ are indicated in the figure. The inset shows the Bridgman gasket assembly.

Figure 2: Hole concentration as a function of pressure for different samples: $(\bullet$ and $\square)[\mathrm{N}]=0.5 \%,(\nabla)[\mathrm{Sn}]=0.5 \%,(\triangle)[\mathrm{Sn}]=0.2 \%,(\square)[\mathrm{Sn}]=0.1 \%$ and (o) $[\mathrm{Sn}]=0.05 \%$.

Figure 3: Pressure dependence of the hole mobility in several samples: $(\bullet)[\mathrm{N}]$ $=0.5 \%,(\nabla)[\mathrm{Sn}]=0.5 \%,(\triangle)[\mathrm{Sn}]=0.2 \%,(\square)[\mathrm{Sn}]=0.1 \%$ and $(\circ)[\mathrm{Sn}]=$ $0.05 \%$. The solid lines are the results of our calculations. Curves 1 and 2 represent the homopolar phonon and LO polar phonon contributions, respectively. Curve 3 represents the ionized impurity contribution as calculated for N-doped GaSe.

Figure 4: Pressure dependence of $E_{a}$. Curves labeled with $\mathrm{N}, \mathrm{Sn}_{1}$ and $\mathrm{Sn}_{2}$ correspond to the nitrogen and tin levels, respectively. The solid lines illustrate the theoretically calculated dependence of $E_{a}$ according with the proposed model. The inset show a schematic comparison of the Coulomb potential and the proposed impurity potential model. 


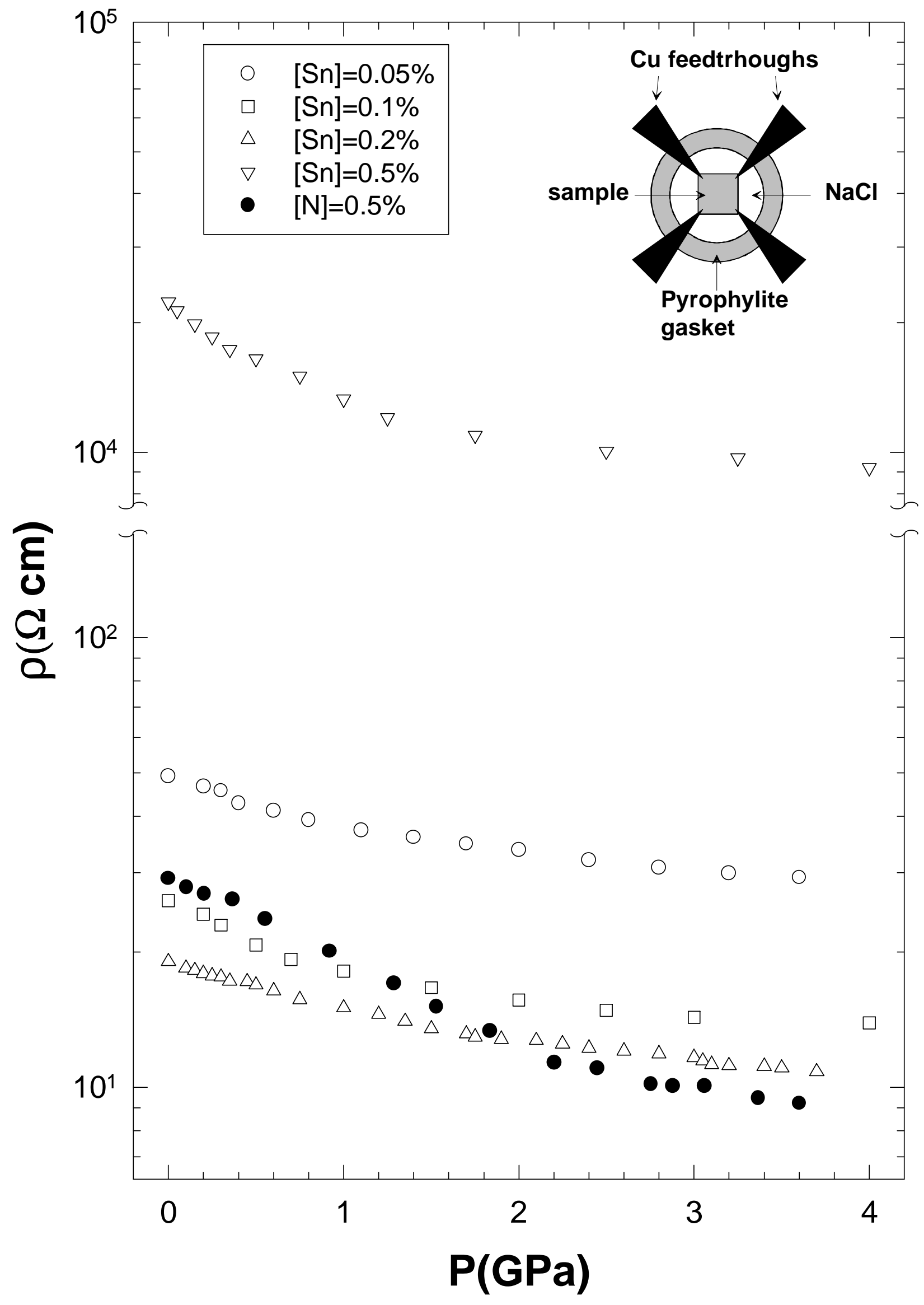




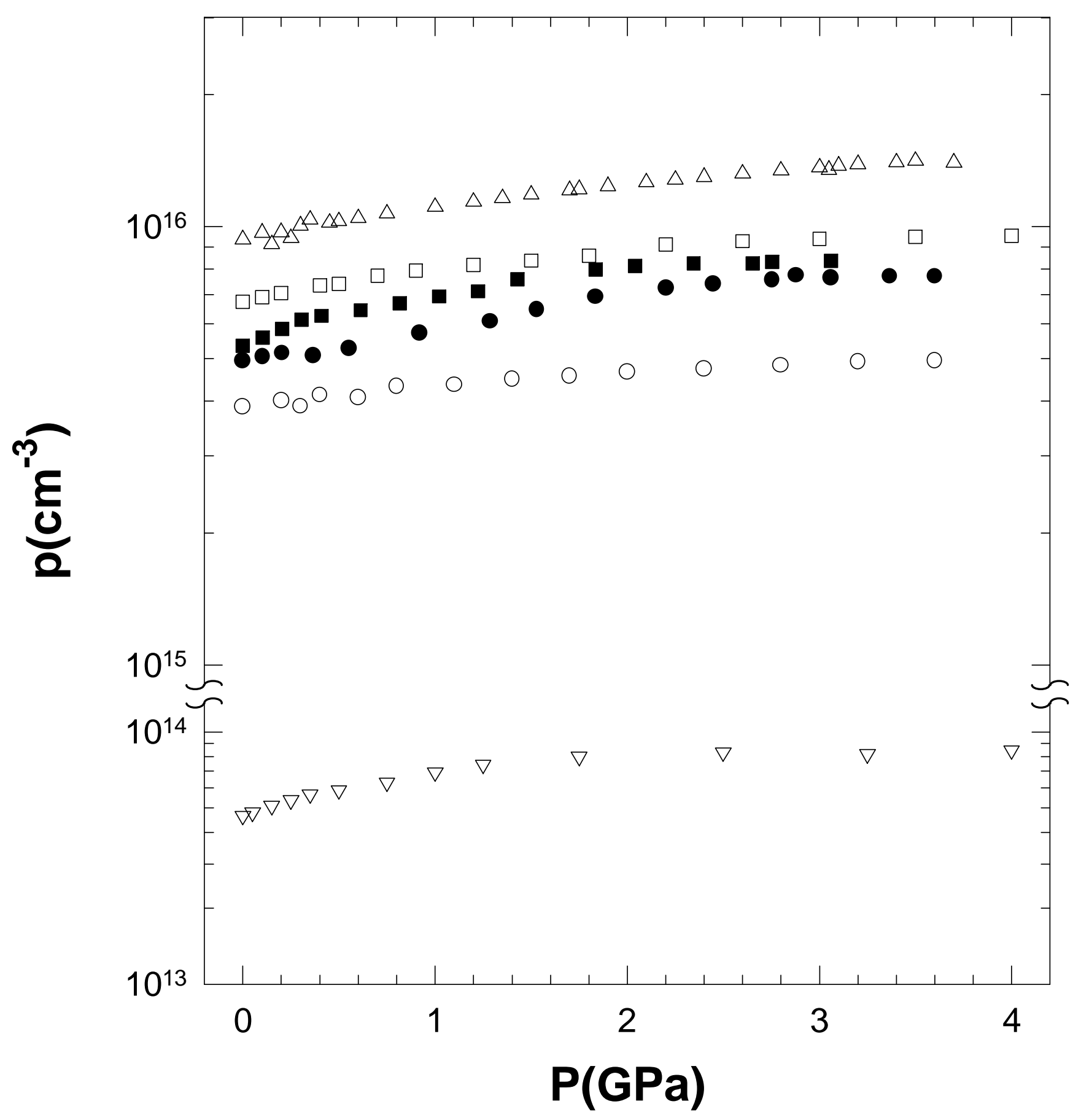




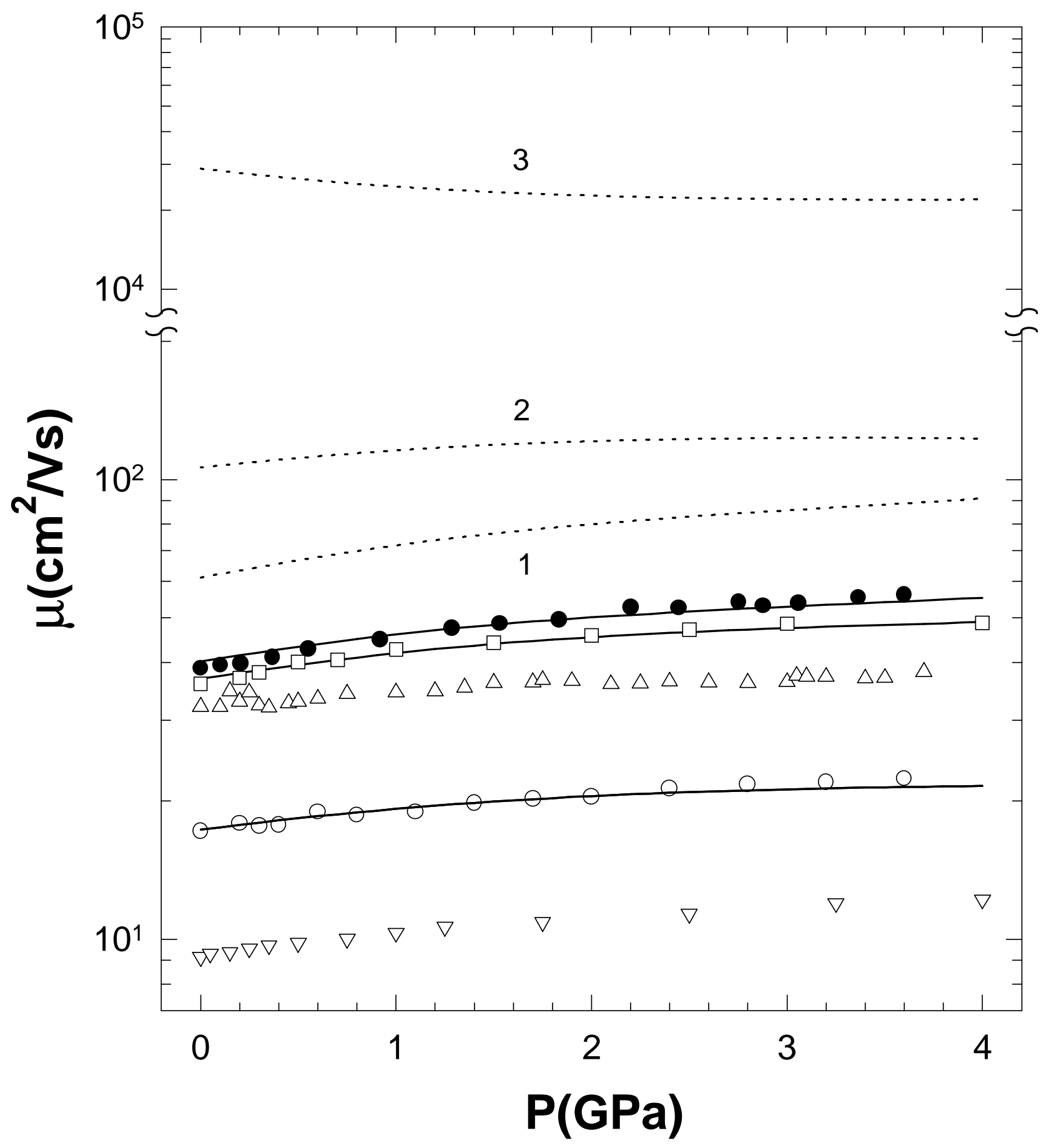




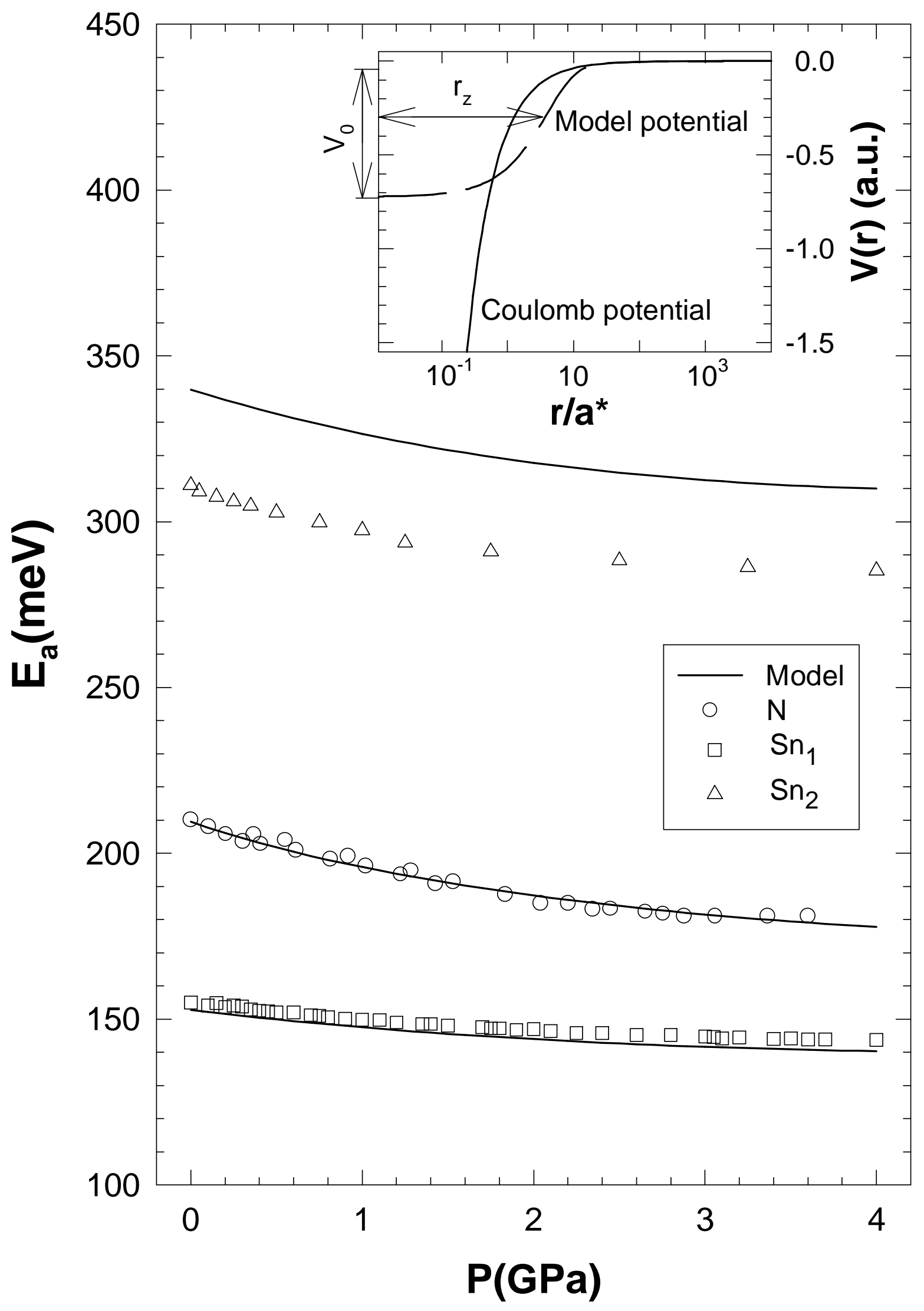

